\title{
Numerical investigation into the influence of mould shape on the pulverulention of pellet
}

\author{
Shen Jiangfei1, a, Wu Kai1, b , Cui Xian'an 1, c and Sun yu1 \\ 1School of mechanical engineering, Nanjing University of science and technology, Nanjing 210094, \\ China; \\ a13770964063@163.com, bwukai@mail.njust.edu.cn,c446730184.@qq.com
}

\section{Keywords: Pellets, FEM, Powder compaction, Mold optimization}

\begin{abstract}
Pellet has advantages of convenient transportation and improving powder performance, so it has been widely used in biomass-energy, feed industry, fertilizer and waste disposal industry. Low pulverulent ratio is the main target of high quality pellets, therefore FEM method was used to predict the influence of mould shape on the pulverulention of pellet. The fundamental principle of Drucker-Prager Cap model which material properties are relative density dependent was discussed in this article. Numerical investigation into the influence of moulds shape on the pulverulention of pellet was conducted in this article. The simulation results show that the pellets compressed by the moulds with concave arc type or small angle oblique line type hole have better outer wall surfaces than convex arc type and large angle oblique line type hole. The powder density of the pellets surface can be increased dramatically through changing the taper hole structure to multistage taper hole. The structure optimization of moulds can significantly improve the pulverulent ratio of pellets.
\end{abstract}

\section{Introduction}

Pellets are a notably form of powder in the feed, biomass fuel, fertilizer, waste treatment and other industries. Pellets have numerous advantages over other forms, such as their low transport cost, long storage life, mechanical and chemical stability, ease of use and well usability. So pellets has been widely used in powder compaction area. Pellets are generally manufactured by ring die or flat die pellets mill, powders which have been disposed are compressed into the hole of the die, and powders will be compressed into a column through a short time pressure maintaining. Pellets can be obtained by cutting off the columns. The pellets compacted by die have many advantages, such as high production efficiency, low energy cost and the smooth surface of pellets [1].

Thorough research, many methods for analyzing the compression of powder have been developed. The most three popular methods are DEM [2] (discrete element method), slab method [3] and FEM [4] (finite element method ). Those works do lots of jobs in simulating the real action of powders. DEM model is the most visualized model in simulating the powder action, in this model powders was figured as a single sphere or polyhedron, and the contact force, friction force and shear force between powders have specific rule dependent on the powder type. The DEM model has well accuracy of powders, but the calculation of the model often can not been accepted, especially when simulate powder compaction. And also it would be difficult achieve accurate results when the powders have remarkable shape [5,6]. Slab method is a simplification of FEM model, it often be used in simulate roller compaction, and this model has great advantage in calculation time. But this model often can not achieve good accuracy. Lots experiments and theoretical researches showed that the mechanical characteristics of powders compaction process has great similarity with ordinary solid medium $[5,6]$. This means the yield characteristics and plastic surface theory which have been widely used in analyzing the solid medium can be used in powder compaction. So its a great idea to simulate powder compaction process by considering the powders as continuous medium, and build the theory of elastic-plastic deformation and constitutive model. DPC (Drucker-Prager Cap) model is the most famous model appiled to powder compaction FEM simulation. Powders will show great different with different relative density. Cunningham [5] build the single hardening parameter model which can describe the change law of powder mechanical property with relative density approximately, but 
this model does not reflect the real property of powder. So density-dependent model was researched by Tuhin Sinha [7], he also simulated the powder compaction process, and experiment verification. Zhu feilong [8] simulated the powder rolling process in ring die mill with FEM method, researched the pressure distribution of the inter surface of ring die. Those researches have great significance to explore the powder compaction process, they will do great help in researching the numerical investigation into the influence of mould shape on the pulverulention of pellet.

Pellets compacted by ring die or flat die often have large pulverulent ratio during transportation, and this is not allowed especially medical pills or biomass pellets. So lots research of optimization of the ingredients were conducted by scholars, but those were limited by the use field of pellets, and have difficulties in conduction. The mould shape is the key factor to form high quality pellets, so this paper focused on introducing the numerical investigation into the influence of mould shape on the pulverulention of pellet.

The DPC model and density dependent model of powders were introduced in this article. The simulation methods and subroutine procedure was discussed in this article, and the mould shape was optimized for high quality pellets

\section{Drucker-Prager Cap model}

\section{Basic principle introduction.}

DPC model is a typical model of double yield surface model, which is widely used in medical compaction, cosmetics and geotechnical analysis industry. The curves of the yield surface of DPC model in the $p-q$ space is shown in Fig.1, where $p$ is the hydrostatics stress, and $q$ is the Mises equivalent stress. Shear failure surface Fs and volume yield surface $F C$ are the main surface of the yield surface curves. In order to facilitate computer calculation, the smooth surface that named $\mathrm{Ft}$ was established in order to connect the shear failure surface Fs and volume yield surface $F C$ smoothly.

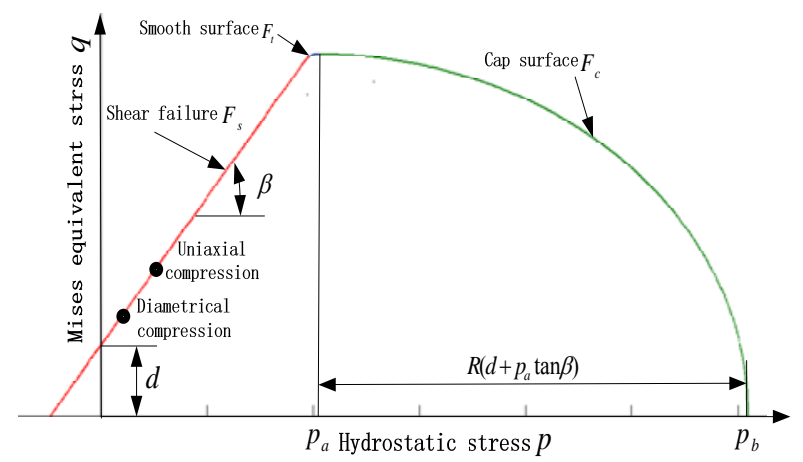

Fig. 1 Yield surface of DPC model

Shear failure surface $F S$ is the yield surface of plastic shear flow, the deformation caused by volume expansion is "inflation" phenomenon cut [9] . Eq. 1 is the shear failure surface function expression :

$$
F_{s}(p, q)=q-p \tan \beta-d=0
$$

Where $\beta$ is the internal friction angle, $d$ is the cohesion, $p=(1 / 3)^{\sigma}$ is the hydrostatics stress, and $q=\sqrt{(3 / 2)(s: s)}$ is the Mises equivalent stress. $s$ is the tensor of deviator stress.

There is no real physical meaning of the smooth surface, its function expression is as the Eq. 2

$$
F_{t}(p, q)=\sqrt{\left(p-p_{a}\right)^{2}+\left[q-\left(1-\frac{\alpha}{\cos \beta}\right)\left(d+p_{a} \tan \beta\right)\right]^{2}}-\alpha\left(d+P_{a} \tan \beta\right)=0
$$

Where $p_{a}=\frac{p_{b}-\mathrm{Rd}}{1+R \tan \beta}$ is the evolution parameter that determines the compaction, depend on the volume of plastic deformation, $\mathrm{Pb}$ is the hardening parameter, $\alpha$ is the shape parameter of the smooth surface, its general value is between 0.01-0.05 [10]. 
The 'cap' surface represents an ellipse with a constant eccentricity in the p-q plane, it is a volume yield surface, as expressed in Eq. 3.

$$
F_{c}(p, q)=\sqrt{\left(p-p_{a}\right)^{2}+\left(\frac{R q}{1+\alpha-\alpha / \cos \beta}\right)^{2}}-R\left(d+P_{a} \tan \beta\right)=0
$$

Where $\mathrm{R}$ is the cap shape parameter.

\section{Measurement of DPC model}

The mechanical properties of the powders will change dramatic during the process of compression, even all the parameters of the yield surface model will be changed when the powder density change. Due to the mechanical properties of the powders and density is closely related. Therefore, it is necessary to introduced relative density as the state variables of the material parameters, relative density of powders is refers to the powder density divide the density that solid phase rate is 1 , the expression is as Eq. 4:

$$
\gamma=\frac{\rho}{\rho_{\text {full }}}
$$

Where $\rho$ is the density of test sample, $\rho_{\text {full }}$ is the density of powders that solid phase rate is 1 .

The measurement of the accurate parameters of DPC model should be done on the three axis pressure tester. It is a long, expensive process, and often limited by the powder type. In order to simplify the determination process of measuring the DPC model parameters, scholars do lots work to study the DPC model principle. And found out that the model parameters can be obtained by simple multiple tensile and compression tests with a single hole die. Although this test method will increase the test time, but the measurement process is simply, easy to execute and low cost.

Young's modulus E and Poisson's ratio $v$ of elastic parameters can be determined by two time cylindrical die hole extrusion tests with upper and lower pressure head. The effect of the friction force on the hole wall can be eliminate by coated with lubricate. Fig. 2 is a typical diagram of axis stress vs axial strain and axial stress vs radial stress of single axial cylindrical powder compaction process. In the Fig. 2, the unloading section is not completely linear, but the section of BD is approximate linear, so, in order to achieve simplified model, the section of BD can be used as the calculating parameters. The elastic parameters of $E$ and $v$ can be measured by testing figures, it can be calculate by Eq. 5 with the unloading curve of BD section in Fig. 2a and Fig. 2b [11].

$$
\begin{aligned}
& \frac{E(1-v)}{(1+v)(1-2 v)}=\frac{d \sigma_{z}}{d \varepsilon_{z}} \\
& \frac{1-v}{v}=\frac{d \sigma_{z}}{d \sigma_{r}}
\end{aligned}
$$

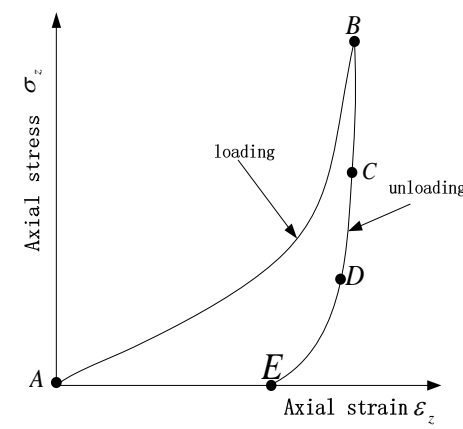

a. Axis stress vs axial strain

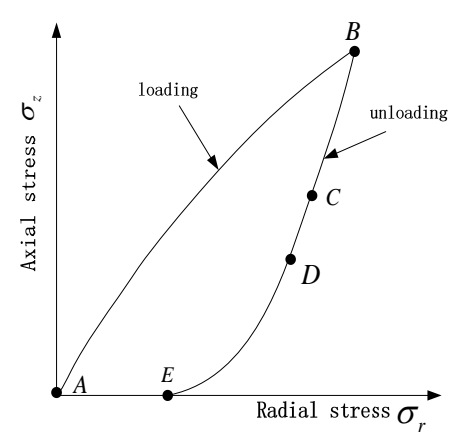

b. Axial stress vs radial stress

Fig. 2 Evolution of powder stress state during compaction

The shear yield surface in the p-q plane is a straight line. So, that means the shear yield surface can be determined by two special points. Therefore the parameters of friction angle $\beta$ and cohesive force $\mathrm{d}$ can be measured by two simple tests. And as showing in the Fig. 1, the two tests are the radial compression test and axial compression test. The radial compression test needs to produce certain shape thin disks of the corresponding density, and test the disks by brazilian disk splitting method. The brazilian disk splitting method is a classical method to determine the tensile failure stress, and 
the equivalent tensile stress $\sigma_{d}=\frac{2 F_{D}}{\pi D h}$, where FD is pressure force, $\mathrm{D}$ is the diameter of disks, $\mathrm{h}$ is height of disks. The axial compression test needs to produce cylindrical powder sample of the corresponding density, and test the sample with crush test method. The crush stress is $\sigma_{c}=\frac{4 F_{c}}{\pi d^{2}}$, where $F_{C}$ is compaction force, $d$ is the diameter of samples. There the the parameters of friction angle $\beta$ and cohesive force $\mathrm{d}$ can be obtained by Eq. 6 and Eq. 7 .

$$
\begin{aligned}
& \beta=\tan ^{-1}\left[\frac{3\left(\sigma_{c}-d\right)}{\sigma_{c}}\right] \\
& d=\frac{\sigma_{c} \sigma_{d}(\sqrt{13}-2)}{\sigma_{c}-2 \sigma_{d}}
\end{aligned}
$$

The shape of volume yield surface is like a cap, it can be determined by $R$ and $P_{a}$. The parameters can be measured by axial extrusion test in a single hole die. The powders will be yield when loading to the point of B as shown in Fig. 2. So, according to the theory of Cunningham [5] and Shang. [6], the cap surface parameters $R$ and $P_{a}$ can be obtained by Eq. 8 and Eq. 9 .

$$
\begin{aligned}
& R=\frac{\sqrt{6}}{3} \sqrt{\frac{\left(1+\alpha-\frac{\alpha}{\cos \beta}\right)^{2}\left(p_{B}-p_{a}\right)}{q_{B}}} \\
& p_{a}=-\frac{\left[3 q_{B}+4 d \tan \beta(1+\alpha-\alpha / \cos \beta)^{2}\right]}{4(1+\alpha-\alpha / \cos \beta)^{2}}+\frac{\sqrt{9 q_{B}^{2}+24 d q_{B}(1+\alpha-\alpha / \cos \beta)^{2} \tan \beta+8\left(3 q_{B} p_{B}+2 q_{B}^{2}\right)(1+\alpha-\alpha / \cos \beta)^{2} \tan ^{2} \beta}}{4(1+\alpha-\alpha / \cos \beta)^{2}}
\end{aligned}
$$

Where $p_{B}=\frac{\sigma_{z}^{B}+2 \sigma_{r}^{B}}{3}$ is the hydrostatics stress of point $\mathrm{B}$, and $q_{B}=\left|\sigma_{z}^{B}-\sigma_{r}^{B}\right|$ is the Mises equivalent stress of point $\mathrm{B}, \sigma_{r}^{B}$ is the radical stress of the point $\mathrm{B}, \sigma_{z}^{B}$ is the axis stress of point $\mathrm{B}$.

\section{Simulation method of powder compaction}

The difficulties of the powder compaction simulation are the changes of yield surface and large deformation. The parameters of the yield surface would change constantly with the relative density of powders. The relative density of powders will changing constantly in the compression process, so the mechanical parameters will change constantly. But the single hardening parameter $P_{b}$ of modified DPC model in Abaqus can not reflect the real characters of powders. So the equivalent model [5] of Cunningham can not achieve accurate results in powder compaction simulation. Therefore subroutine must be used in order to change the parameters of elements before every step. VUSDFLD is the subroutine of abaqus which can realize the change of the parameters. The abaqus software can read the equivalent volumetric strain after every incremental step, and refresh the DPC model parameters according to the relative density of previous step before each step. The equivalent plastic strain variable is PEQC, and according to the Gurson [13] theory, the relative density can be obtained by the equivalent plastic strain by Eq. 10. Therefore, the material parameters database can be built up by Eq. 10 and the material parameters of the relative density. Then refresh the model parameters of elements according to the material parameters database and previous step analysis results.

$$
\rho=\rho_{o} \exp \left(-\varepsilon_{\text {vol }}^{p l}\right)
$$

The elements would be distorted seriously during the process of the simulation of the powder compression, and thus causing the computation stop. ALE adaptive mesh technology has fully absorbed the advantages of Lagrangian finite element and Eulerian finite element. The ALE technology has been widely used in fluid mechanics. It has been an effective method for solving large deformation simulation, such as fluid mechanics and material processing process and so on [12]. In order to avoid building large part of powders and complex calculation, thus Euler boundary was used in the simulation, the materials were flow in from the up part and flow out from the down part. The 
comprehensive use of ALE technology and Eulerian finite element simulation technology can greatly improve the convergence of the finite model, improve the accuracy of the simulation results

\section{FEM Model}

The finite element simulations were simulated using the commercial software ABAQUS v6.13. ABAQUS has built-in the DPC constitutive model for modeling powders and other porous materials. The material properties of powders was PH102 which is taken from shang.DC [6]. A single hole die was used to compress pellet, the diameter of the hole is $3 \mathrm{~mm}$, and effective length is $20 \mathrm{~mm}$, the length of the tapered bore is $5 \mathrm{~mm}$, and the tangent of the angle is 0.2 . The elements type of the model is CAX4RA which is a four-node, two-dimensional and axi-symmetric elements. The friction of the interface of the powder and tooling is constant, and its value is 0.2 . The compression speed is $100 \mathrm{~mm} / \mathrm{s}$.

ABAQUS does not automatically account for the variation in the material parameters occurring due to the evolution of the relative density. Therefore to account for those changes, a Fortran subroutine was used in the FEM simulation in order to modify the material parameters before each time step based on the current local relative density of the elements. So, the subroutine VUSDFLD is used to refresh the elastic and plastic material properties before each time step. At each time step, the subroutine obtains the current strain increments, calculates the local relative density in each element, and then updates the elasto-plastic material properties in the corresponding elements based on the table data provided during property definition. The flowchart describing the main program and the subroutine is presented in Fig. 3.

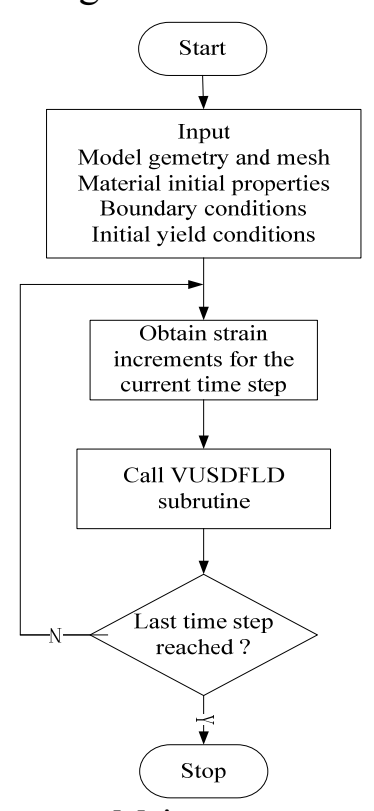

a. Main program

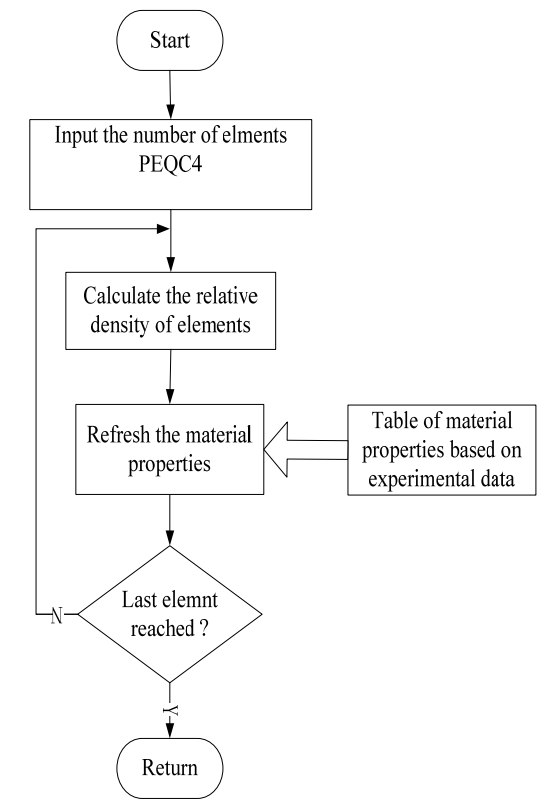

b. VUSDFLD subroutine

Fig. 3 Flowchart of the user-defined subroutine and main program

\section{Results and discussion}

The tapered bore shape of die is the key factor of the pellets density distribution, and the density distribution is one of the main reasons leading to pulverization. The most commonly used types of hole die is small angle oblique line, large angle oblique linear, convex arc and concave arc. The density distribution cloud map of pellets compressed by four type of hole die are presented in Fig. 4. PEQC4 is the bulk plastic strain of elements, and the relationship of the volume plastic strain and relative density is shown as Eq. 10, therefore the PEQC4 value can be used to reflect the relative density of the powders. As it is shown in the pictures, the density of the central part of pellets are much higher than the outside wall. The most uniform density distribution pellet was compressed with the die with convex arc. The density of the material near the outside wall reduced sharply, and the 
plastic strain of the outside wall of pellet is even positive value, this means the powders in the outside wall were not compressed. Therefore the pellets compressed by convex arc type hole die would be easily damaged. The density distribution of the pellet compressed form oblique line type hole die shown higher quality than convex arc type hole die. And the more smaller angle of the tapered bore, the more higher quality of the pellets. The density distribution of the pellet compressed form concave arc type hole die shown the most highest quality, the powder distribution is relatively symmetrical, and the powder density of the outside wall is also higher than others. So in order to enhance the pellets' ability of resisting damage, concave arc type hole die is preferable.
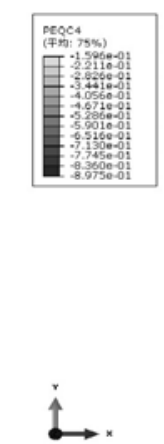
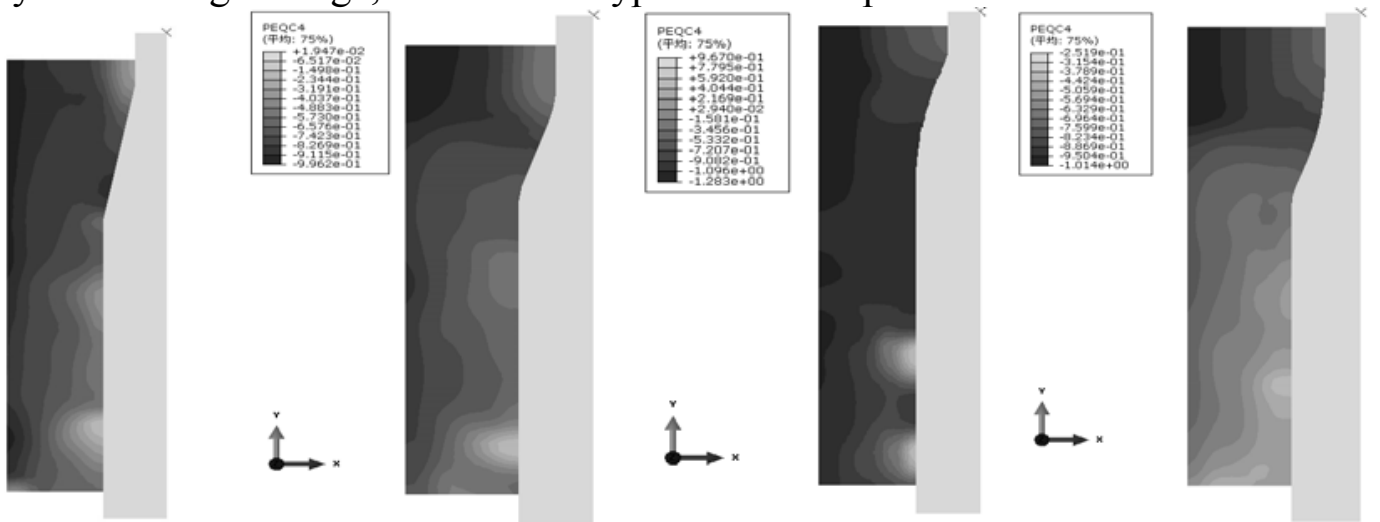

a. Small angle oblique line b. Large angle oblique linear c. Convex arc

Fig. 4 Density distribution cloud map of pellets

Although the concave arc type hole die shows better performance in manufacture pellets, but the powder density of the outside wall of pellets still too low to resist the damages which would happen during manufacturing, packaging and shipping. Therefore, the die need to be optimized for enhancing the hardness of the pellets without adding other material and energy waste. According to the density distribution of pellets in Fig. 4 and lots of simulation results, the die can be optimized as Fig. 5. The optimized die is multi-section tapered bore die, and the total length of the multi-section tapered bore is same with the non-optimized die.
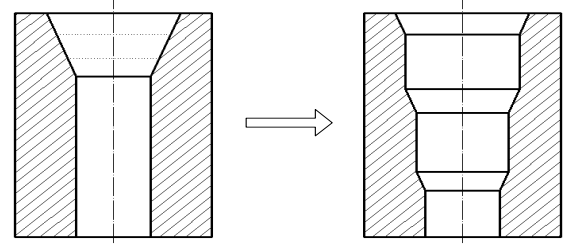

Fig. 5 Optimization of tapered bore

The density distribution cloud map of pellets compressed by multi-section tapered bore die were showed in Fig. 6. The results show that the powder density of the outside wall of the pellets increased greatly. The pellets compressed by multi-section can behave more hardness to resist the damages. There the pulverulent ratio could be reduced by using the optimized die.

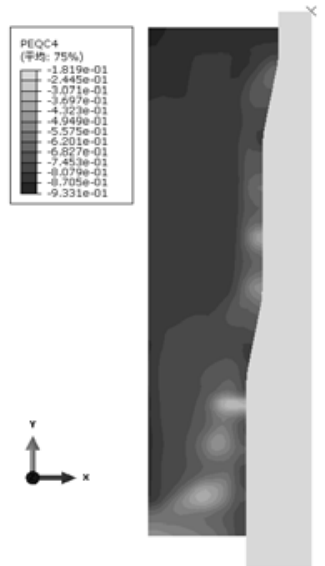

a. Double section tapered bore

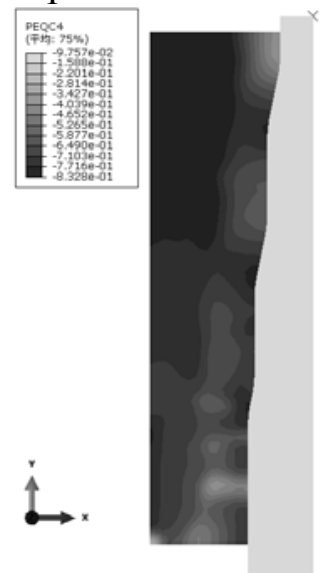

b. Three section tapered bore

Fig. 6 Density distribution cloud map of pellets compressed by optimized die 


\section{Summary}

A density-dependent DPC model simulation method was discussed in this article with considering the relationship of powder material mechanics parameters with relative density. The ALE adaptive element, euler element technology and subroutine simulation methods were used to build up the model in abaqus software. Numerical investigation into the influence of moulds shape on the pulverulention of pellet was conducted in this article. The simulation results show that the pellets compressed by the moulds with concave arc type or small angle oblique line type hole have better outer wall surfaces than convex arc type and large angle oblique line type hole. The powder density of the pellets surface can be increased dramatically through changing the taper hole structure to multistage taper hole. The structure optimization of moulds can significantly improve the pulverulent ratio of pellets.

\section{References}

[1] Wu kai, Sun Yu. Pellet Molding Technology and Equipment. Beijing: Science Press, 2013.

[2] Li F., Pan J. and Sinka I.C. Contact laws between solid particles. Journal of the Mechanics and Physics of Solids. 2009, 57: 1194-1208.

[3] D. Kumar, U.S. Dixit. A slab method study of strain hardening and friction effects in cold foil rolling process [J]. Journal of Materials Processing Technology, 2006, 171: 331-340.

[4] H. Diarra, V. Mazel, A. Boillon et al. Finite Element Method (FEM) modeling of the powder compaction of cosmetic products: Comparison between simulated and experimental results. Powder Technology, 2012, 224: 233-240.

[5] Cunningham J.C. Experimental studies and modelling of the roller compaction of pharmaceutical powders. Philadelphia: Drexel University, 2007.

[6] Chenglong Shang. Modelling powder compaction and breakage of compacts. Leicester: Doctor of Philosophy at the University of Leicester, 2012.

[7] Tuhin Sinha, Jennifer S. Curtis, Bruno C. Hancock, Carl Wassgren. A study on the sensitivity of Drucker - Prager Cap model parameters during the decompression phase of powder compaction simulations. Powder Technology. 2010,198: 315-324.

[8] Zhu Feilong, Wu kai, Ding Wuxue et al. Finite Element Simulation of Powder Rolling Process in Ring Die Pellet Mill. China powder science and technology, 2013, 19 (6): 1-4.

[9] Wu, C.Y., Ruddy,O.M, Bentham.A.C, Hancock.B.C, Best.S.M, Elliott.J.A. Modelling the mechanical behaviour of pharmaceutical powders during compaction. Powder Technology. 2005, 152: $107-117$.

[10]Fei Kang. Application of Abaqus in geotechnical engineering. Beijing: China Water Power Press, 2010.

[11]L.H. Han,, J.A. Elliott, A.C. Bentham, A. Mills,G.E. Amidon, B.C. Hancock. A modified Drucker-Prager Cap model for die compaction simulation of pharmaceutical powders. International Journal of Solids and Structures. 2008, 45: 3088-3106.

[12]Ding Junhong, Jin Xianlong, Guo Yizhi et al. Study on 3_D numerical simulation for soil cutting with large deformation. Journal of agricultural machinery, 2007, 38(4): 118-121.

[13] Gurson A.L. Continuum theory of ductile rupture by void nucleation and growth: part I, yield criteria and flow rules for porous ductile media. Journal of Engineering Material and Technology. Transactions of the ASME. 1977, 99: 2-15. 